\title{
Birgit Mack: \\ Der Deutsche Dublin-Core-Tag 1999 am 28. Oktober in der Deutschen Bibliothek Frankfurt
}

Ziel dieser Tagung war, ein breites Publikum über die Dublin-Core-Metadaten-Anwendungen in Deutschland zu informieren und neue Entwicklungen vorzustellen.

Zunächst aber ein paar Worte über Sinn und Zweck von Metadaten:

Metadaten sind Daten über Daten; d.h. es sind übergeordnete Daten zu Medien bzw.

Informationseinheiten (auch visuelle Dokumente, Audio-Objekte ...).

Metadaten beschreiben ein bestimmtes Medium zum Zweck des Wiederfindens in Katalogen

(z.B. konventionelle Zettelkataloge) oder Datenbanken.

Durch das Internet wachsen die digitalen Informationsangebote explosionsartig an. Mit Hilfe von Metadaten, die Internetsuchmaschinen berücksichtigen, sollen diese Internetquellen suchbar gemacht werden.

Dublin Core ist ein System zur einheitlichen Erschließung von Internetressourcen mit standardisierten Metadaten. Es ist interdisziplinär und hat sich nahezu weltweit durchgesetzt. Beim Deutschen Dublin-Core-Tag wurde deutlich, dass es etliche deutsche Projekte aus verschiedenen Bereichen (Bibliotheks-, Archiv-, Verlagswesen ...) gibt, die sich mit Dublin-CoreMetadaten beschäftigen.

Eines davon ist META-LIB, das von der DFG gefördert wird. Teilnehmer sind die Staats- und Universitätsbibliothek Göttingen, Die Deutsche Bibliothek, die Bayerische Staatsbibliothek und das Deutsche Bibliotheksinstitut. Seit kurzem beteiligt sich auch das Bibliotheks-ServiceZentrum Konstanz mit der Aufgabe, eine geeignete Suchmaschine zu entwickeln.

META-LIB hat zum Ziel, Richtlinien für den Einsatz von Metadaten (insbesondere Dublin-CoreMetadaten) zu entwickeln, um digitale und digitalisierte Ressourcen in wissenschaftlichen Bibliotheken zu erschließen.

Beeindruckend an den anderen vorgestellten Projekten (Malvine, Stabi Berlin, MathNet, Uni Osnabrück) ist, dass sie sehr schnell in anderen Ländern Fuß gefasst haben, z.B.: Teilnehmer von Malvine sind Länder wie Frankreich, Spanien, Portugal ...

Auch Verlage beschäftigen sich verstärkt mit Metadaten im elektronischen Publizieren. Ein Referent des Springer-Verlags beschrieb das verlagseigene Metadaten-Format DOI-X, das allerdings einige Unterschiede zu Dublin Core aufweist.

Die Deutsche Bibliothek beteiligt sich an der Entwicklung einer eindeutigen, dauerhaften Identifizierung von Internet-Ressourcen, sogenannte Persistent Identifier (URLs ändern sich ja häufig). DDB beabsichtigt, die Aufgaben einer nationalen Agentur zur Vergabe und Verwaltung der Identifier zu übernehmen.

An diesem Tag wurde deutlich, dass viele deutsche Bibliotheken die Notwendigkeit sehen, sich mit Metadaten zu beschäftigen, und dass es dabei eine große Bereitschaft gibt, international zusammenzuarbeiten.

\section{Internetadressen zu Metadaten bzw. Dublin Core:}

